\title{
FPGA Implementation for Rapid Prototyping of High Performance Voltage Source Inverters
}

\author{
Yukun LUO, M A AWAL, Wensong YU, and Iqbal HUSAIN
}

\begin{abstract}
Field-programmable gate array (FPGA) is a powerful platform that can play an essential role in high-performance digital control of power electronics systems. However, the FPGA system's design is quite different from that of a traditional microprocessor or a digital signal processor (DSP). Instead of sequential programming using high-level languages, such as $\mathrm{C} / \mathrm{C}++$, FPGA controller implementation requires a hardware description language (HDL) such as Verilog and VHDL, which requires extensive verification and optimization during the design process. This paper proposes a systematic FPGA design methodology with optimum resource utilization for rapid prototyping of high-performance power electronics applications to facilitate the widespread adoption of FPGA technology in power electronics. The FPGA controller design is concurrent with the power stage and utilizes high-level synthesis (HLS) tools and Simulink code generation toolbox. This paper covers the detailed design, implementation, and experimental validation of two specific applications, i.e., an active power filter (APF) and a motor emulator (ME), demonstrating the generalized features of the methodology. Employing fundamentally different control structures, both application examples achieve ultra-high current control bandwidth leveraging SiC MOSFETs switching at no less than $100 \mathrm{kHz}$.
\end{abstract}

Index Terms-Field-programmable gate array (FPGA), model predictive control, voltage source inverter, wide band gap device.

\section{INTRODUCTION}

A $\mathrm{S}$ the field-programmable gate array (FPGA) becomes more accessible and powerful due to the advance in integrated circuit technology, the FPGA application in power electronics is becoming popular. FPGA's concurrent nature and powerful computation resources make it more suitable for some applications over the traditional microcontrollers and digital signal processors (DSPs). The detailed comparison between DSP/microcontroller and FPGA is presented in [1]. Sensorless motor drive control using FPGA was presented in [2]. Computationally heavy control method like model predictive control (MPC) [3]-[7] was also implemented in FPGA. Due to multiple $\mathrm{I} / \mathrm{O}$ pins and logic resources, FPGA is very suitable

Manuscript received January 6, 2021; revised March 30, 2021; accepted May 7 , 2021. Date of publication December 30, 2021; date of current version December 24, 2021. This work was supported by the Office of Energy Efficiency and Renewable Energy (EERE), U.S. Department of Energy, under Award Number DE-EE0006521 with North Carolina State University, Power America Institute.

All authors are with North Carolina State University, 1791 Varsity Dr, Raleigh, North Carolina 27695, United States.

Digital Object Identifier 10.24295/CPSSTPEA.2021.00030 for applications that require complex modulation strategies [8], [9].

The adoption rate of wide-bandgap devices, such as Silicon Carbide (SiC), is steadily increasing in power electronics with the advances in manufacturing processes. The faster switching capability of wide-bandgap devices than that of Si devices provides an opportunity to improve control bandwidth and poses challenges in device protection. A FPGA-based short-circuit protection circuit for the $10-\mathrm{kV} \mathrm{SiC}$ MOSFET is proposed in [10]. To fully utilize the high switching frequency capability to boost the control performance, the FPGA-based high-performance digital control platform provides a better choice since the increased switching frequency poses a limitation on the allowable latency for the digital system, including the sampling and control algorithm computation delay. In [11], high bandwidth VIENNA rectifier with over $1 \mathrm{MHz}$ switching frequency was achieved using a FPGA based control platform.

FPGA-based system development using hardware description language (HDL) is a rigorous and cumbersome process. Fortunately, FPGA manufacturers have introduced high-level design tools, such as high-level synthesis (HLS) [12], and block diagram based design toolbox, such as System Generator for DSP from Xilinx and DSP Builder from Altera, to help expedite the development process. A critical step to accelerate the development process is to efficiently incorporate these high-level design tools into the existing design workflow in power electronics. Examples of previous work on the FPGA controller development process include predictive control in FPGA design using HLS [3], resource streaming design technique in motor drive applications [5], and block diagram based FPGA design [13]. In [14], detailed design and implementation of an impedance identification algorithm block for resonant inverter using HLS were presented. In this work, the digital control platform chosen is the SoC type (ARM+FPGA) in order to bring more flexibility in the system design and deliver high bandwidth, low latency power electronic controllers. This paper presents the design methodology using a toolchain involving multiple software applications while illustrating the merit of this type of platform. Two examples are provided with different implementation strategies. Compared to other FPGA implementation papers [15], [16] which focus on only specific function block or design tool, this paper provides a more systematic high-level perspective and methodology for designing digital systems requiring high control bandwidth, high fidelity modeling, and low latency for rapid prototyping of power elec- 
TABLE I

Comparison Between Different Type of FPGA Platform

\begin{tabular}{|c|c|c|c|c|}
\hline & Type 1 & Type 2 & Type 3 & Type 4 \\
\hline Architecture & Pure FPGA & $\mathrm{FPGA}+\mathrm{DSP}$ & SoC & Third party solution \\
\hline Toolchain & Native & Native & Native & Third party software \\
\hline Merit & $\begin{array}{l}\text { 1. Simple one chip solution. } \\
\text { 2. Highly customizable. }\end{array}$ & $\begin{array}{l}\text { 1. Highly customizable. } \\
\text { 2. } 28 \text { Abundant peripherals. }\end{array}$ & $\begin{array}{l}\text { 1. Simple one chip solution. } \\
\text { 2. Highly customizable. } \\
\text { 3. Abundant peripherals. }\end{array}$ & $\begin{array}{l}\text { 1. High level graphic development } \\
\text { tool is easy to learn. } \\
\text { 2. No need for low level FPGA } \\
\text { design knowledge. }\end{array}$ \\
\hline Demerit & $\begin{array}{l}\text { 1. The lack of peripherals. } \\
\text { 2. Require knowledge on } \\
\text { low level FPGA design. }\end{array}$ & $\begin{array}{l}\text { 1. Hardware design is } \\
\text { complicated. } \\
\text { 2. Require knowledge on } \\
\text { low level FPGA design. }\end{array}$ & $\begin{array}{l}\text { 1. Require knowledge on } \\
\text { low level FPGA design. } \\
\text { 2. Logic resource may be } \\
\text { limited. }\end{array}$ & $\begin{array}{l}\text { 1. Hardware is not customizable. } \\
\text { 2. Performance of the FPGA } \\
\text { may be limited by the third } \\
\text { party development software. }\end{array}$ \\
\hline
\end{tabular}

tronics applications.

The proposed rapid prototyping approach not only maximizes the FPGA resource utilization, but also can be naturally integrated into the existing design workflow of power electronics applications. Moreover, this paper divulges how to assign various power electronics digital control tasks, like ADC, reference frame transformation, control algorithm, PWM generation, and communication, to different parts (ARM core or FPGA) of the SoC platform according to system-level priority requirement, latency requirement, and implementation complexity. Two specific examples with detailed implementation and decision-making for each function block are presented. The first example illustrates a high bandwidth active power filter (APF) capable of compensating up to 51st harmonic utilizing an interleaved $\mathrm{SiC}$ voltage source inverter (VSI). The second example is a high bandwidth motor emulator (ME) utilizing a SiC VSI, which can emulate the high-frequency ripple current caused by the inverter's switching under test (IUT).

The rest of the paper is organized as follows: In Section II, FPGA platform selection and design toolchain are introduced. In Section III, the proposed design methodology is presented. In Section IV, APF example with detailed implementation and experimental results are covered. In Section V, the ME example is illustrated with implementation details and experimental results. Finally, conclusions are drawn in Section VI.

\section{FPGA PlatForm \& ToOLCHAIN}

There are quite a few ways to use FPGA in real-time control systems. In Table I, four types of FPGA digital system architecture are listed and compared. Type 1, denoted as pure FPGA architecture, is the bare FPGA structure that does not provide the peripherals and requires low-level design knowledge. Type 2 solution combines the abundant peripherals of DSP with the powerful FPGA [17]; however, building a fast and reliable communication link between DSP and FPGA could be pretty challenging. Furthermore, the two-chip solution complicates the controller board design. Type 3 is the FPGA-embedded general-purpose processor system-on-chip (SoC) platform, which typically includes many peripherals, like CAN, USB, Ethernet and UART, and analog-to-digital converter. These peripherals can make the platform more flexible and save FPGA's logic resource for computational use compared with Type 1 architecture. Third-party FPGA solution is denoted as Type 4. Companies, such as National Instruments, provide their hardware consisting of a FPGA chip from Xilinx or Altera and their own high-level block diagram-based development software such as LabVIEW FPGA. This solution provides an easier learning curve for FPGA programming but has several limitations. First, this platform's flexibility is limited since the hardware cannot be customized to achieve specific functionality. Second, the FPGA performance might not be fully utilized since the third-party software's code optimization is usually subpar compared to that of the native development tools.

In this work, we opt for the Type 3 implementation using the ZYNQ-7000 family from Xilinx for efficient development and effective resource utilization. The ZYNQ-7000 Systemon-Chip (SoC) integrates a dual-core ARM Cortex-A9 based processing system (PS) and $28 \mathrm{~nm}$ Xilinx programmable logic (PL) in a single device. This solution provides better communication performance between processor and FPGA and simplicity of controller board design over Type 2 solution using separate DSP and FPGA chips. A PicoZed system on module (SoM) board (XC7Z015-1SBG485) from Avnet is used for design simplicity. It is worth noting that the selected board is not the most powerful option from the PicoZed family. The selected option provides $3.3 \mathrm{~V} \mathrm{I} / \mathrm{O}$ voltage level whereas more powerful and faster devices can only support $1.8 \mathrm{~V}$ or $2.5 \mathrm{~V} \mathrm{I}$ $\mathrm{O}$ voltage levels. I/O pins with lower voltage levels have lower noise immunity. In power electronics applications, especially with fast switching SiC devices, signal noise is a big concern, and hence, the selection is made for better noise immunity.

The development toolchain used for ZYNQ-7000 SoC in this work includes Xilinx Vivado, Xilinx software development kit (SDK), Vivado High-Level Synthesis (HLS), and System Generator for DSP. The Vivado is for the system-level synthesis and implementation of the FPGA hardware design. The Xilinx SDK is used to program the ARM core (PS) within the ZYNQ-7000 SoC. The Vivado HLS is used for designing the FPGA IP core using $\mathrm{C} / \mathrm{C}++$. Last but not least, System Generator for DSP is a block diagram-based MATLAB Simulink toolbox into which HDL and HLS designs can be im- 


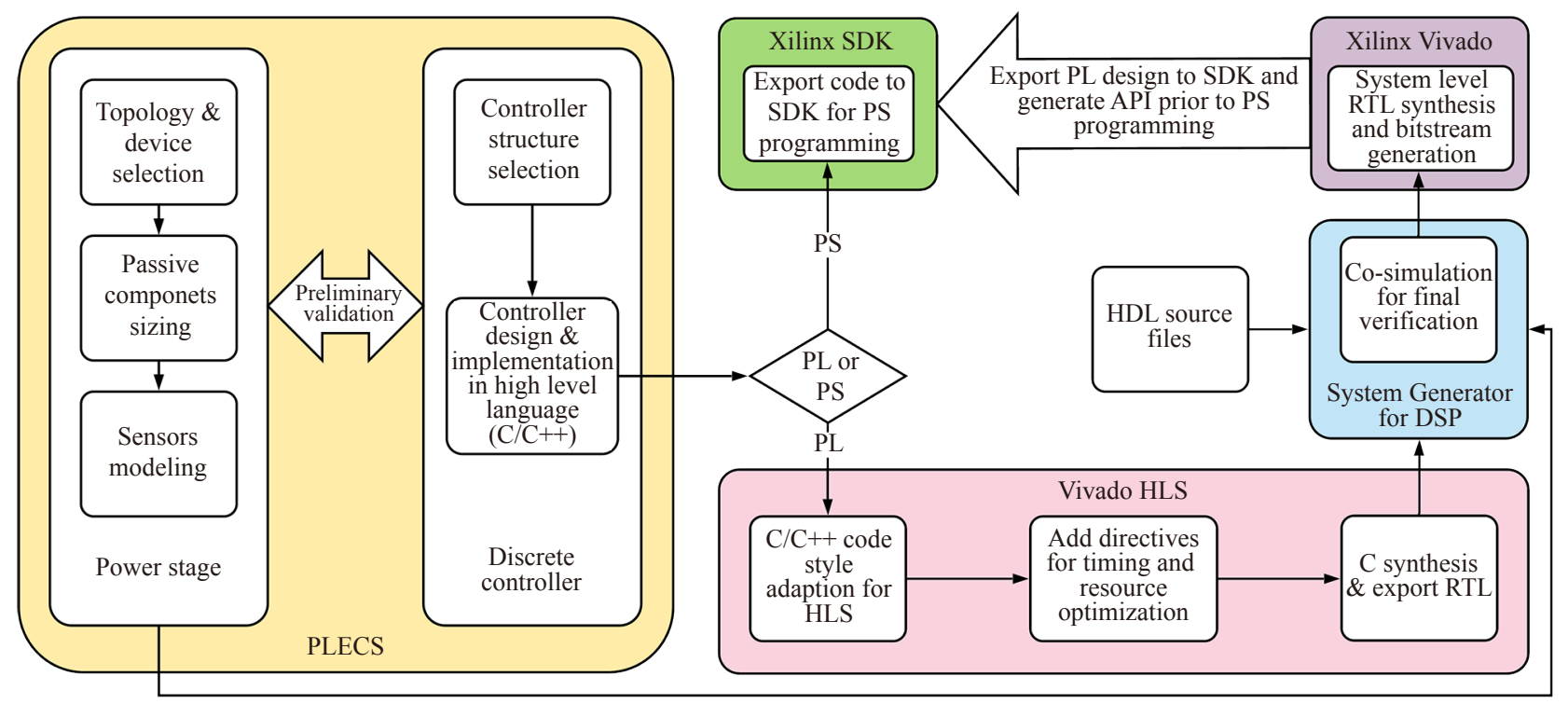

Fig. 1. Workflow of proposed FPGA design methodology for rapid prototyping in power electronics.

ported. It should be pointed out that other FPGA manufacturers have similar toolchains for the same purpose.

\section{Design Methodology}

The workflow of the proposed FPGA design methodology for rapid prototyping digitally controlled power electronics system is shown in Fig. 1. The design methodology emphasizes the concurrent design of the power stage and digital controller, which enables high-level system performance and optimum resource utilization. The power stage's basic specifications include topology, power device performance, passive component values, and sensor performance. These hardware specifications could also affect the digital control system's design, such as the controller structure, controller parameters, and control iteration speed. Using software like PLECS and Simulink, the power stage can be accurately represented by the circuit diagram. Simultaneously, the function blocks like C-Script and S-Function could simulate the C code-based digital controller's behavior. This way, the preliminary system specifications can be established.

After the preliminary round of design iteration, we should have a functional prototype $\mathrm{C}$ code for the digital controller. The next question is whether to implement the control algorithm in PS or PL cores. The PS CPU is versatile and easy to program using high-level languages. However, the PS's sequential nature means the absolute performance (algorithm execution time) for a specific task is no match to the PL. Although there are HLS tools to simplify FPGA's design process, it is still more complicated than the PS design. Also, the hardware resource of FPGA would limit how many algorithms can be executed concurrently. Resource utilization and clock speed are trade-offs. If the resource utilization is very high, there is less placing and routing flexibility, resulting in slower clock speed, with techniques like loop unrolling, loop merge, loop flattening, pipelining, and resource sharing, FPGA clock speed,

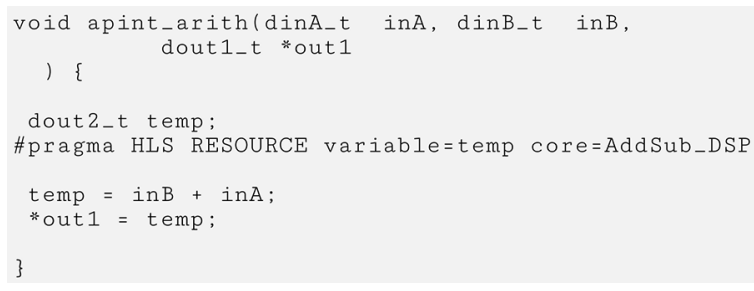

Fig. 2. Example code snippet with HLS pragma for resource utilization optimization [18].

and latency can be improved. Even though the latency may increase slightly, the total execution time could be reduced with faster clock speed. With the help of tools like HLS, those optimization techniques can be easily applied using corresponding directives [18]. Whereas using the conventional FPGA design method, the exploration of the design space might not be sufficiently rigorous. In Sections IV and V, two examples of the detailed decision-making between PL and PS are presented.

For implementation in PS, this prototype code can be directly applied with minor modification. However, if the algorithm is to be implemented in PL, HLS would be a good choice. The Xilinx HLS tool synthesizes a C function into an IP block to integrate into a hardware system. It is tightly integrated with the rest of the Xilinx design tools and provides comprehensive language support, and features for creating the optimal implementation for a $\mathrm{C}$ algorithm [18]. The design flow of HLS contains the following stages:

1) Compile, execute, and debug the $\mathrm{C}$ algorithm.

2) Synthesize the $C$ algorithm into an RTL implementation, optionally using user optimization directives.

3) Generate comprehensive reports and analyze the design.

4) Verify the RTL implementation using a push button flow.

5) Package the RTL implementation into a selection of IP formats. 

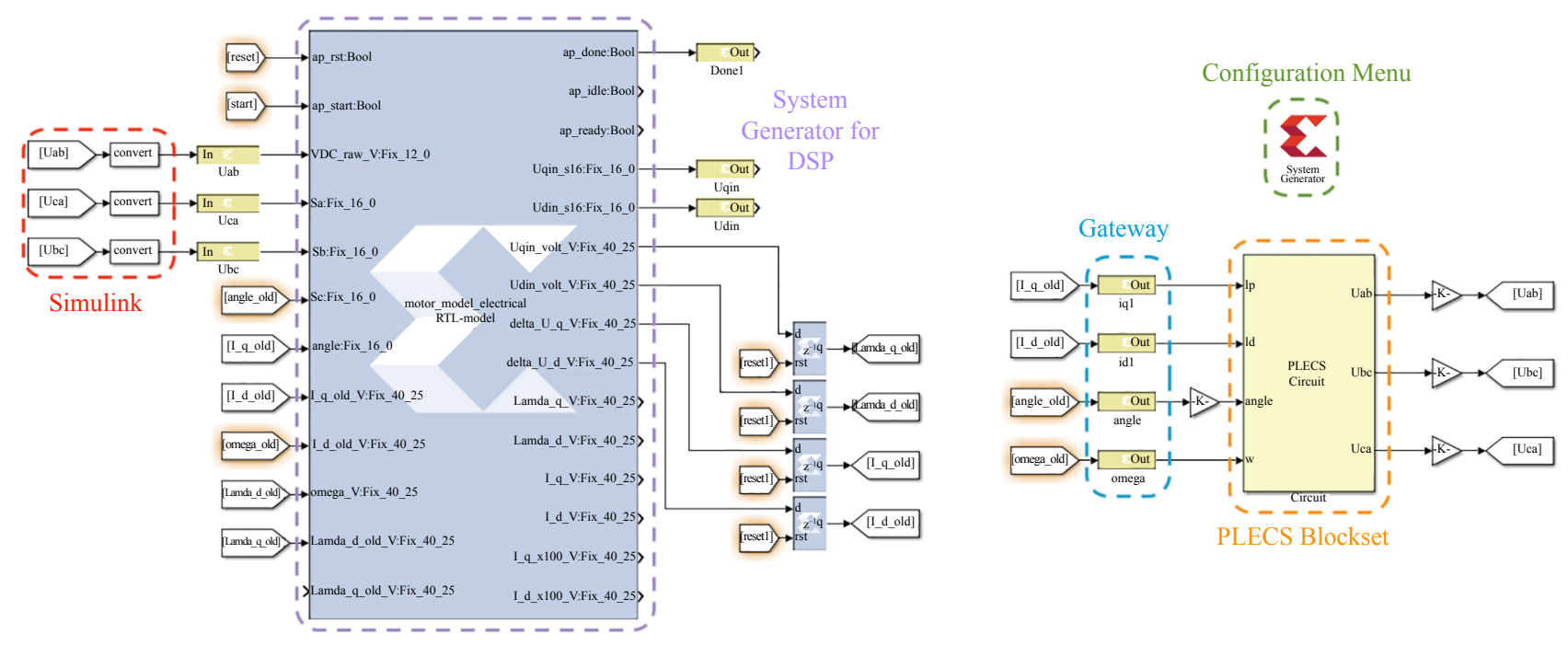

Fig. 3. Co-simulation setup in Simulink environment, with the source for different blocks labelled.

In this paper, to better tailor this design flow for power electronics applications, stages 4 and 5 are replaced by System Generator for DSP toolbox. In stage 1, the adaption of code style is needed for better results. For example, avoiding too many arithmetic operations within one line of code could improve resource utilization, as discussed in Chapter 3 of [18]. After the code style optimization, the next step is to add directives to define device constraints, and resource utilization preference, described in stage 2 , such as setting the clock speed and using DSP core to implement a multiplier [18]. In Fig. 2, an example code snippet with pragma directive to select DSP core for summation operation is presented. More information regarding how to apply directives and pragma can be found in Chapter 1 of [18]. Moreover, different interfaces, like AXI4 and RAM, can be added to HLS design using corresponding directives. In stage 3, timing, latency, and resource utilization estimation are provided after $\mathrm{C}$ synthesis in a few minutes. This feature significantly accelerates the performance optimization process of HLS design.

Another critical step in the workflow is the functional verification of the HLS design. In [14], a C-based test bench was preferred for fast verification of the design in HLS, referred to as stage 4. However, for power electronics application, System Generator for DSP toolbox is preferred for verification purpose in this paper. System Generator for DSP is a design tool in the Vivado Design Suite that enables the MathWorks model-based Simulink design environment for FPGA design. Previous experience with Xilinx FPGA devices or RTL design methodologies is not required when using System Generator. Designs are captured in the Simulink modeling environment using a Xilinx-specific block set. Downstream FPGA steps, including RTL synthesis and implementation, are automatically performed to produce a FPGA programming bitstream [19]. Design in M-code, HDL code, and C/C++ code (HLS) can be imported to the System Generator environment for simulation and verification using MCode, Black Box, and Vivado HLS block, respectively, described in Lab 2 of [19]. As mentioned

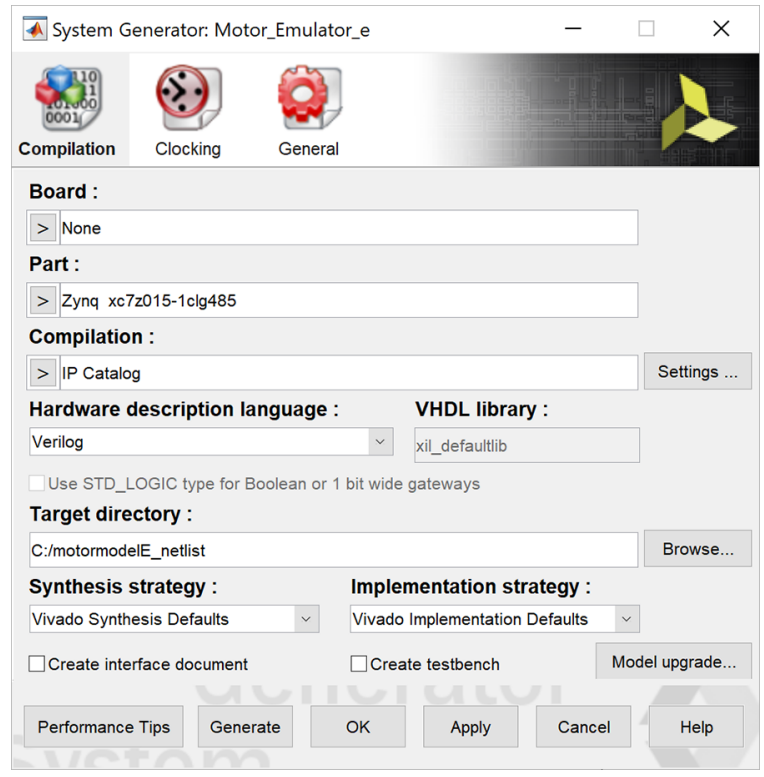

Fig. 4. The configuration menu in System Generator for DSP can be accessed by double-click the red Xilinx icon in Fig. 3. The target device, system constraints, and export preference can be defined in this menu.

before, the power stage is modeled in the PLECS environment. With the PLECS blockset, the PLECS model can be imported to the Simulink environment. Therefore, the co-simulation between digital control algorithm, modeled in System Generator, and power stage, modeled in PLECS blockset, can be easily carried out in Simulink. The screenshot of the Simulink cosimulation setup is shown in Fig. 3. In Fig. 3, the blocks with white background are native Simulink blocks, enclosed in the red rectangle. The blocks with yellow background, enclosed in orange rectangle, are from PLECS blockset, in which power stage can be modeled. The blocks with Xilinx icon, enclosed in purple rectangle, are from System Generator for DSP toolbox. It is worth noting that the gateway blocks in blue rectangle and the configuration menu icon in the green rectangle are also 
TABLE II

COMPARISON OF INTERNAL AXI INTERFACE

\begin{tabular}{lccc}
\hline \hline Name & AXI4-Lite & AXI4 & AXI4-Stream \\
\hline Implementation Complexity & Low & Medium & High \\
Address Format & Memory-mapped & Memory-mapped & No address \\
Throughput & Low & Medium & High \\
Overhead & Low & Medium & High \\
Targeted Application & Mainly for IP core control purpose & For bulk data transmission & For image and video processing application \\
\hline \hline
\end{tabular}

from System Generator toolbox. The gateway blocks are used to define the boundary of HDL design. While in the configuration menu, the target device, system constraints, and export preference can be established. The detail of this menu is shown in Fig. 4. Admittedly, the C-based simulation in HLS is faster than the co-simulation in Simulink. However, with the existing power stage model in PLECS, the co-simulation method can be integrated into the traditional design workflow of power electronics more naturally and is still faster than the conventional RTL-based design verification [14]. Furthermore, with the fully functional prototype $\mathrm{C}$ code, a high number of design iterations are not expected in the co-simulation stage.

With the successful verification, the System Generator for DSP can package the design as an intellectual property (IP) core and export it to Vivado for system-level RTL synthesis and bitstream generation. The System Generator export setting can be accessed in the menu by double-clicking the Xilinx logo in Fig. 3. In the menu presented in Fig. 4, one can set the target device and clock speed and the IP export directory. More details regarding this menu setting can be found in Lab 3 of [19]. Once the setting is finalized, the IP can be generated at the designated directory by hitting the "Generate" button. To use the IP in Vivado, the IP directory should be added to the IP catalog. With all the necessary IPs added to Vivado, system-level design can be synthesized and implemented, followed by the bitstream generation.

Then the bitstream can be exported to Xilinx SDK. Once the hardware design is transferred to the SDK environment, the board support package (BSP) with all the peripheral driver application programming interface (API) is generated. Finally, the application software for the ME system can be developed. The Xilinx SDK comes with great example designs for almost all the APIs. Since the Xilinx ZYNQ-7000 SoC platform selected for the examples of this paper contains a dual-core ARM processor (PS) and the FPGA (PL), there is more flexibility in the digital system design. The PS system development is quite similar to that of a DSP or microprocessor and beyond the scope of this paper. However, the PS and PL's interconnection is essential for system performance and should not be ignored. The ZYNQ-7000 device provides a powerful internal bus called AXI4 (Advanced eXtensible Interface 4) to transfer data between the PL and the PS. There are three variations of the AXI interface for different application purposes. The comparison of the three variations is presented in Table II. The detailed decision-makings for each function block and AXI interface are illustrated in the examples provided in Section IV and Section V.

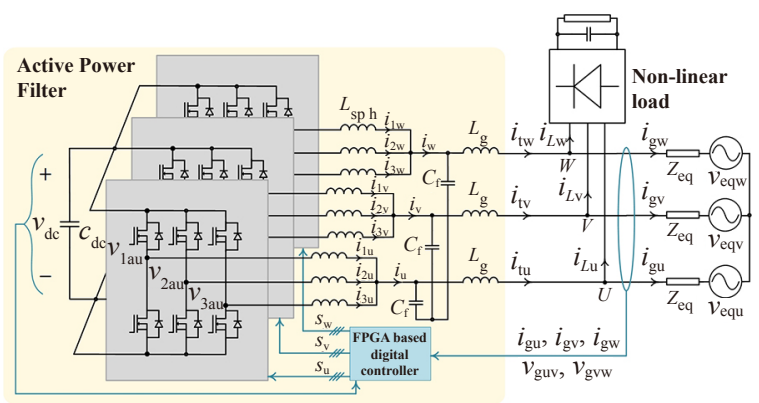

Fig. 5. Active Power Filter (APF) architecture.

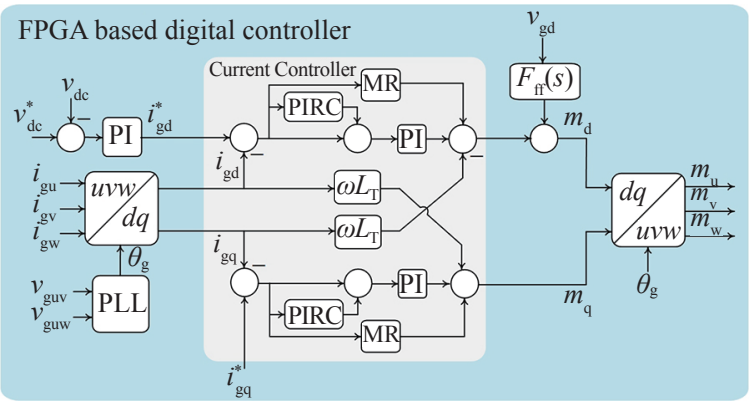

Fig. 6. APF multiple resonant-plug in repetitive controller (MR-PIRC) current controller structure.

\section{Active Power Filter (APF) Example}

Active power filter can eliminate the grid current harmonics by actively compensating harmonic currents required by nonlinear loads. A shunt APF utilizing interleaved SiC VSI and high-performance FPGA platform is presented in this section with implementation details. The topology of the interleaved APF architecture is presented in Fig. 5. The interleaving enables the reduction of passive component sizes of the LCL filter. The current controller for the APF is a hybrid combination of multiple resonant (MR) and plug-in repetitive (PIRC) compensators represented in Fig. 6. The developed APF is rated for $125 \mathrm{kVA}$ operation and can compensate up to 51st order of harmonic with effective switching frequency of $300 \mathrm{kHz}$. The APF's rating and parameters are listed in Table III. The detailed controller design is illustrated in our previous work [20].

\section{A. FPGA Based Implementation}

The first decision to be made is whether a subsystem should be implemented in PS or PL. For the APF, the controller is set 
TABLE III

APF RATINGS \& PARAMETERS

\begin{tabular}{lcc}
\hline \hline $\mathrm{S}_{\text {rated }}$ & Rated power & $125 \mathrm{kVA}$ \\
$V_{0}$ & Nominal voltage & $480 \mathrm{~V}$ \\
$\omega_{0}$ & Nominal frequency & $2 \pi(60) \mathrm{rad} / \mathrm{s}$ \\
$f_{\mathrm{sw}}$ & Switching frequency & $100 \mathrm{kHz}$ \\
$f_{\mathrm{s}}$ & Sampling frequency & $100 \mathrm{kHz}$ \\
$v_{\mathrm{dc}}^{*}$ & Nominal DC bus voltage & $860 \mathrm{~V}$ \\
$C_{\mathrm{dc}}$ & DC bus capacitor & $1.5 \mathrm{mF}$ \\
$L_{\mathrm{sph}}$ & Converter-side sub-phase inductor & $40 \mu \mathrm{H}$ \\
$L_{\mathrm{g}}$ & Network-side inductor & $40 \mu \mathrm{H}$ \\
$C_{\mathrm{f}}$ & Filter capacitor & $2 \mu \mathrm{F}$ \\
\hline \hline
\end{tabular}

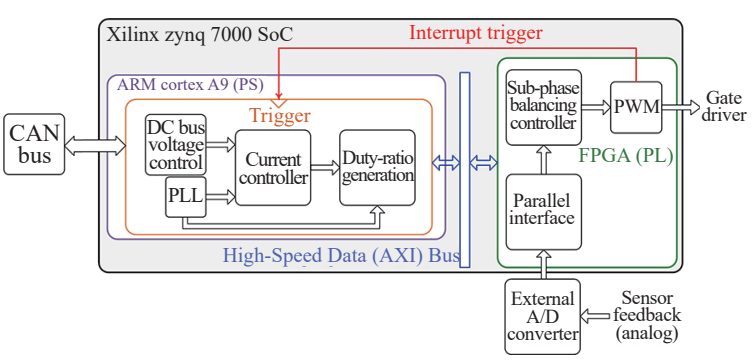

Fig. 7. ZYNQ-7000 design block diagram for APF.

to be iterating at $100 \mathrm{kHz}$. The less computation intense controller structure [20] coupled with relative slow iteration speed makes it possible to implement most of the control algorithm in PS. Also, the parameters' fine-tuning during the experiments could be faster if the controllers are implemented in PS. The exception is the sub-phase current balancing control. The reasons for this decision are twofold: First, the results of the external ADC ICs are obtained in PL, which means AXI interface is needed to transfer the data to the controller on the PS side. As introduced in Table II, the most basic AXI4-Lite interface is suitable for this single shot small data transmission with the low overhead requirement. As presented in Fig. 5, there are 15 quantities needed to be sampled at $100 \mathrm{kHz}$ for the APF controller - three grid currents, nine sub-phase currents, the DC bus voltage, and two phase to phase grid voltages. If the sub-phase current balancing control is implemented in PS, then there will be $108(9 \times 12)$ bits of additional data needed to be transferred from PL to PS in each control period. This would significantly increase the execution time, considering the utilization of the AXI4-Lite bus, and compromise the digital control performance. Second, ideally, the nine sub-phase balancing controllers for each sub-phase should be running concurrently to reduce the latency. The parallelism nature of FPGA makes the PL very suitable for this task.

The overall implementation block diagram for APF is presented in Fig. 7. In addition to the control subsystem, the CAN communication is also implemented in PS. The embedded peripherals, like CAN and Ethernet, make it convenient to run communication tasks in PS. ADC interface, PWM generation, and sub-phase current balancing are assigned to the PL. In this

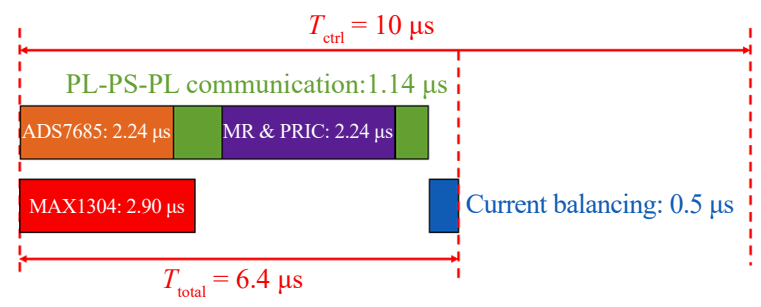

Fig. 8. Task parallelism \& latency analysis for APF.

example, most of the tasks are executed sequentially without much parallelism. The functional blocks in the PL part can be divided into two categories. The sub-phase current balancing block is essentially computation cores. The ADC interface and PWM generator are sequential logic blocks. All the functional blocks except the CAN communication are iterating at 100 $\mathrm{kHz}$.

The sub-phase balancing included nine PI compensators; the implementation of computation cores in PL via HLS is covered in Chapter A of Section V. The concurrent nature of the PL hardware suits the requirements of PWM and ADC interface very well. Moreover, most of the I/O pins of the ZYNQ-7000 $\mathrm{SoC}$ are connected to the PL side. The PWM generation can be implemented with simple logic components such as counters and comparators. The ADC interface design is more complicated due to the large number of signals involved. As mentioned above, there are 15 quantities needed to be sampled at 100 $\mathrm{kHz}$ rate, which exceeds the performance limit of the internal ADC of ZYNQ-7000 SoC. Therefore, four external ADC ICs (two ADS7865 and two MAX1304) are utilized to carry out the sampling task. Both ADC ICs have 12-bit parallel interface for data transmission. Therefore, to save $\mathrm{I} / \mathrm{O}$ pin resources and increase FPGA routing flexibility, all four ICs share one 12bit data bus, but with individual control bus. The increase in latency due to the shared data bus is acceptable considering the relatively slow control frequency of $100 \mathrm{kHz}$. Since all four ICs share the same data bus, one high level state machine is required to coordinate the operation across the ADC ICs. The interface can be programmed either with HDL or System Generator for DSP.

With all the necessary data sampled and transferred to PS, the main current control can be executed. All the computation in PS is executed in floating-point format. The complicated APF controller can finish calculation in time due to the high CPU clock rate of $666.7 \mathrm{MHz}$. Once the control computation is complete, the results are transferred back to PL. In PL, the current balancing controller outputs are added to the results from the main APF controller in PS before the final PWM generation. The sequence and run-time of the designed digital system are shown in Fig. 8.

\section{B. Verification \& Performance Analysis}

As mentioned before, the majority of the control for APF is executed in PS, and the corresponding software is developed using $\mathrm{C}$ code. The controller's verification developed in C can be easily carried out in numerical simulation platforms 


\begin{tabular}{|l|r|r|r|r|}
\hline Resource & Utilization & Available & Utilization \% \\
\hline LUT & 3370 & 46200 & 7.29 \\
\hline LUTRAM & 262 & 14400 & 1.82 \\
\hline FF & 4716 & 92400 & 5.10 \\
\hline BRAM & 0.50 & 95 & 0.53 \\
\hline DSP & 24 & 160 & 15.00 \\
\hline IO & 51 & 150 & 34.00 \\
\hline BUFG & 3 & 32 & 9.38 \\
\hline
\end{tabular}

Fig. 9. Resource utilization report for APF.
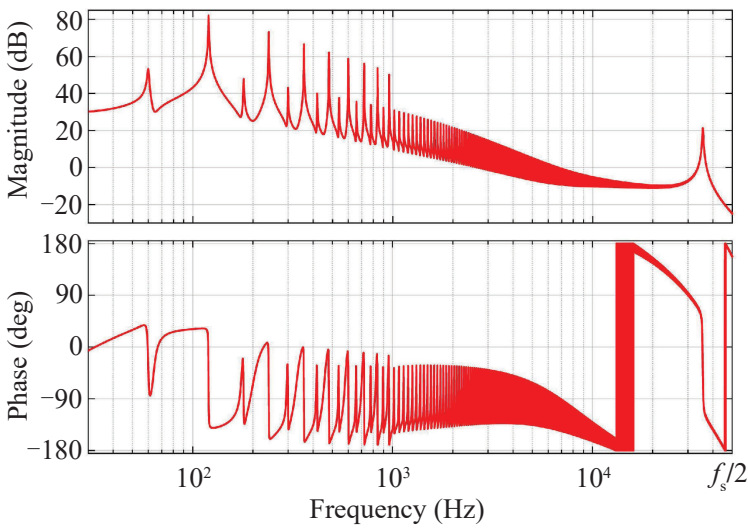

Fig. 10. Compensated open loop response.
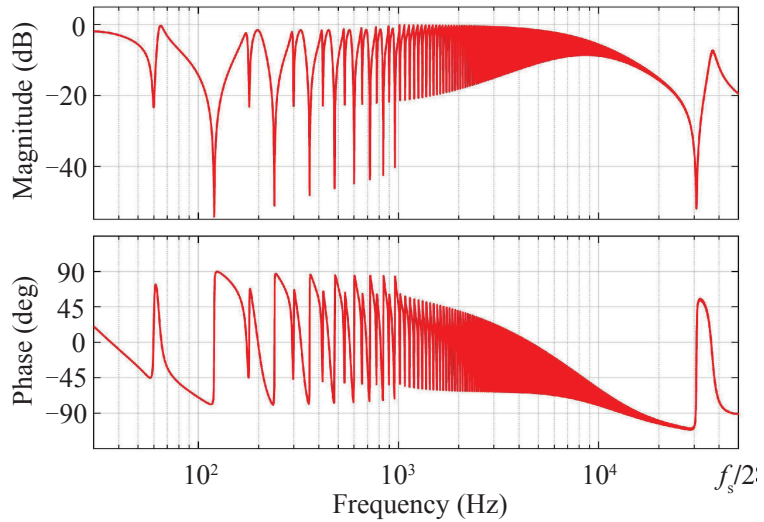

Fig. 11. Converter admittance $Y_{\mathrm{d}}(\mathrm{s})=i_{\mathrm{gd}}(\mathrm{s}) / v_{\mathrm{gd}}(\mathrm{s})=i_{\mathrm{gq}}(\mathrm{s}) / v_{\mathrm{gq}}(\mathrm{s})$.

such as PLECS. The rapid verification method for PL design is covered in Chapter B of Section V. As shown in Fig. 8, the most time-intensive task is the main current control algorithm and the ADC. The delay caused by the data transfer between PS and PL is not significant in this application. The total time required to execute all the tasks is $6.4 \mu \mathrm{s}$. When designing a FPGA-based embedded system, two key performance indicators are clock speed and resource utilization. Faster clock speed means a shorter execution time. However, if the clock speed is too high, the design would have setup or hold timing violation. The FPGA clock is set to be $200 \mathrm{MHz}$. According to the timing analysis, the slowest path still has 0.13 ns slack with no hold violation. The resource utilization report is presented in Fig. 9. The utilization of all the resources is pretty low since most of the computation burden is on the PS. The most utilized resource is the I/O pin; this is due to the large number of gating

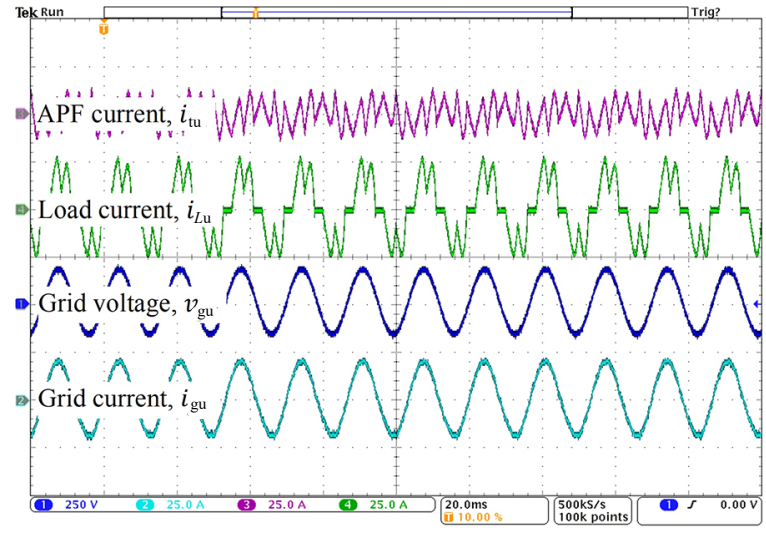

Fig. 12. Steady state operation waveform for APF.

signals and external ADC ICs. However, through the data bus sharing, the utilization is controlled to a relatively low level at $34 \%$.

\section{Controller Analysis \& Experimental Results}

With the proposed controller structure, shown in Fig. 6, and the short sampling time, thanks to the powerful FPGA and proposed design methodology, the system can achieve great transient and steady-state responses and stability irrespective of the grid impedance variation without any active or passive resonance damping measure. The compensated open-loop transfer function $G_{\mathrm{OL}}(\mathrm{s})$ is derived in [20], which includes the non-linearity in real hardware implementation, like implementation delay, the sampling by the digital controller, and the PWM process. From the frequency response of the compensated openloop response $G_{\mathrm{OL}}(\mathrm{s})$ of the current control loop shown in Fig. 10, a gain margin of $\approx 9.3 \mathrm{~dB}$ and a phase margin of $\approx 50^{\circ}$ are achieved despite the high LCL resonance peak.

The converter admittance $Y_{\mathrm{d}}(\mathrm{s})=i_{\mathrm{gd}}(\mathrm{s}) / \nu_{\mathrm{gd}}(\mathrm{s})=i_{\mathrm{gq}}(\mathrm{s}) / \nu_{\mathrm{gq}}(\mathrm{s})$, shown in Fig. 11 , achieves passivity up to $\approx 16 \mathrm{kHz}$. The passive admittance guarantees damping of any resonance caused by uncertain grid impedance in the corresponding frequency range. More discussions regarding controller design and passivity theory based stability demonstration can be found in our previous work [20].

Experiments have been carried out using a full-scale prototype to demonstrate the proposed APF system. This APF is designed for a chiller, which has a diode bridge as the front end. Therefore the non-linear load used in the experiment is a diode bridge. The experimental waveform for steady-state operation is shown in Fig. 12. The FFT results for non-linear load current and grid current is presented in Fig. 13. For a load current of $36.7 \%$ THD, the APF achieves $2.35 \%$ THD in grid current. It can be seen that the harmonic current requested by the non-linear load is supplied by the APF instead of the grid, and up to 51st harmonics are compensated.

Compared with conventional grid-tied LCL converter solutions which require passive [21], or active damping [22] to stabilize the system, the high control update rate in this application, enabled by the powerful FPGA platform and proposed 


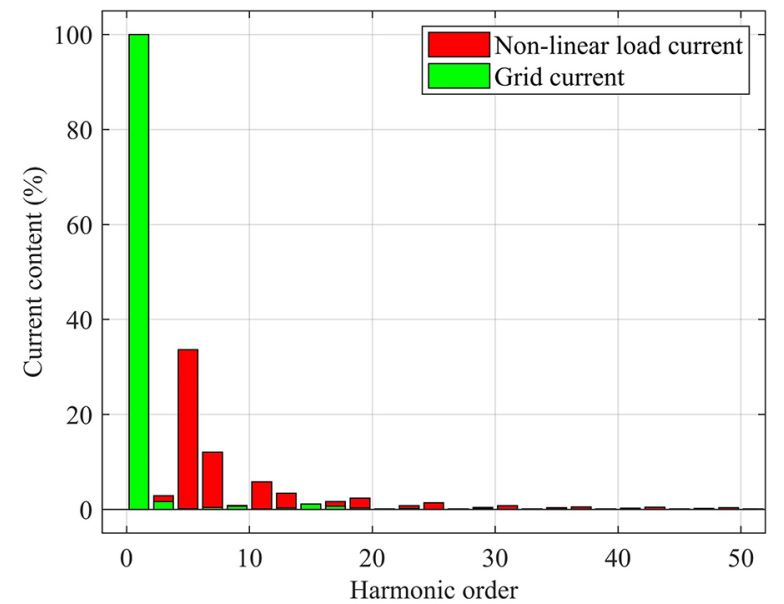

Fig. 13. Spectral content in non-linear load current and in grid current (normalized by fundamental frequency component).

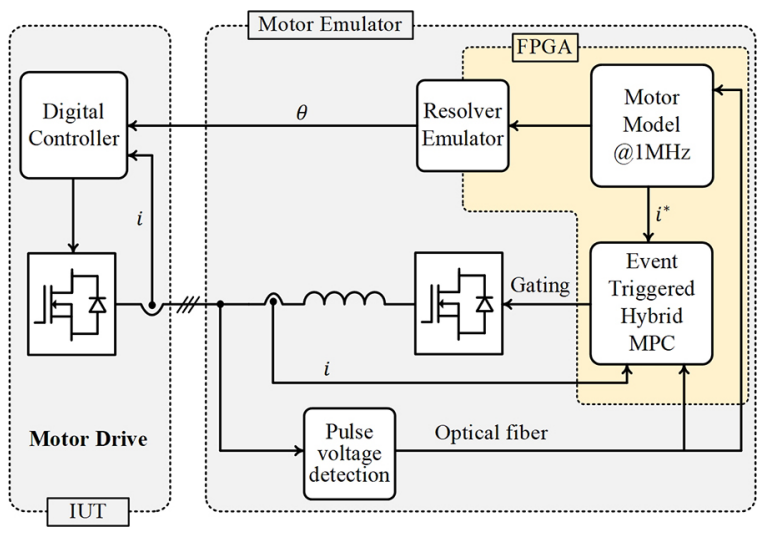

Fig. 14. ME architecture with FPGA implementation overview.

design methodology, eliminates the need for any damping measure.

\section{Motor EMUlator (ME) Example}

Motor emulator (ME) is a power hardware-in-the-loop device that can replicate an electric motor's terminal electrical characteristic. It can be used for testing the motor drive inverter, denoted as inverter under test (IUT) henceforth, without the actual motor and expensive dyno setup. An overview of the system architecture is shown in Fig. 14. The high-performance current controller of the ME is a hybrid combination of integral and finite control set MPC compensators represented in Fig. 15. With two-level VSI topology and a much higher switching frequency than IUT, this high bandwidth ME can emulate high-frequency ripple current caused by the switching of the IUT enabled by the hybrid MPC-based current controller. The ME parameters are listed in Table IV. Furthermore, the controller design details are covered in our previous work [23].

\section{A.FPGA Based Implementation}

As shown in Fig. 14, the computation cores for ME contains high fidelity motor model iterating at $1 \mathrm{MHz}$, a hybrid MPC

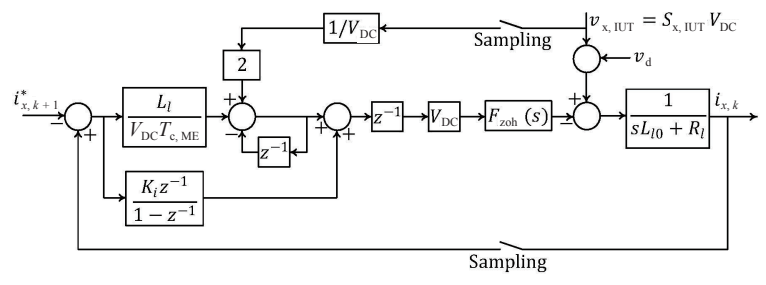

Fig. 15. ME system model with hybrid MPC compensator for small signal analysis.

TABLE IV

ME PARAMETERS

\begin{tabular}{lcc}
\hline \hline$V_{\mathrm{DC}}^{\mathrm{IUT}}$ & IUT DC bus voltage & $300 \mathrm{~V}$ \\
$V_{\mathrm{DC}}^{\mathrm{ME}}$ & ME DC bus voltage & $300 \mathrm{~V}$ \\
$f_{\mathrm{sW}}^{\mathrm{IUT}}$ & IUT switching frequency & $10 \mathrm{kHz}$ \\
$f_{\mathrm{c}}^{\mathrm{ME}}$ & ME control frequency & $400 \mathrm{kHz}$ \\
$L_{\mathrm{l}}$ & Line inductance & $0.15 \mathrm{mH}$ \\
$L_{\mathrm{d}}$ & Target motor inductance & $0.4 \mathrm{mH}$ \\
$L_{\mathrm{q}}$ & Target motor inductance & $0.6 \mathrm{mH}$ \\
$R_{\mathrm{S}}$ & Target motor resistance & $0.1 \Omega$ \\
$\lambda_{\mathrm{pm}}$ & Target motor PM flux & $0.1 \mathrm{~Wb}$ \\
$P$ & Target motor pole number & 8 \\
$t_{\mathrm{DB}}^{\mathrm{IUT}}$ & IUT dead-band time & $2000 \mathrm{~ns}$ \\
$t_{\mathrm{DB}}^{\mathrm{ME}}$ & ME dead-band time & $200 \mathrm{~ns}$ \\
\hline
\end{tabular}

current controller iterating at $400 \mathrm{kHz}$, and resolver emulator iterating at $1 \mathrm{MHz}$. To achieved satisfactory run-time performance, those three tasks should be executed in parallel. Implementing all three computation cores in PL ensures parallelism and eliminated the data transmission requirement between PS and PL for control purposes. Therefore, the entire digital system for ME is implemented in PL, except the Ethernet communication. The Ethernet is mainly for data logging and monitoring purposes. The Ethernet communication between ZYNQ7000 SoC and PC happens every $5 \mathrm{~ms}$, and the PS handles the Ethernet communication. Transferring data from the controller and motor model (located in PL) to the PS is the key to communication. For the ME application, although a small amount of data is generated every $400 \mathrm{kHz}$ or $1 \mathrm{MHz}$, the single-shot AXI4-Lite interface is too slow to finish the transmission within the control period according to the APF result in Fig. 8; a higher performance interface is needed for the ME application. As pointed out in Table II, the higher performance interface has a large overhead. A block memory in PL is utilized as a buffer to bulk the data up for easier high-performance interface implementation to address the issue. AXI4 interface is eventually selected for this application. AXI4-Stream is overkill, and the PL implementation is more complicated. The AXI4 transaction will initiate every $5 \mathrm{~ms}$ to move the data in the buffer to the onboard memory for the PS. Then the Ethernet user datagram protocol (UDP) is initiated to send the data to the PC. This way, the data logging is running entirely in parallel with the control algorithm. The overall implementation block diagram 


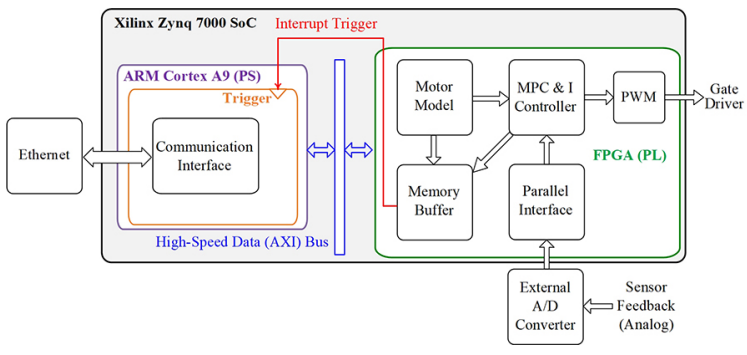

Fig. 16. ZYNQ-7000 design block diagram for ME.

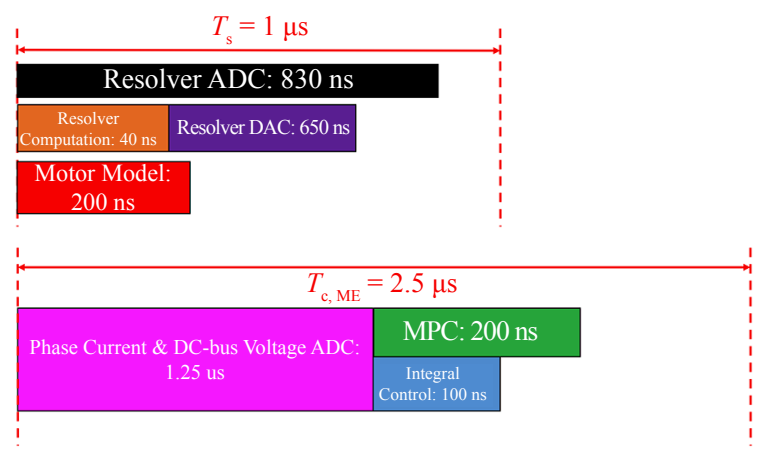

Fig. 17. Task parallelism and latency analysis for ME.

for ME is presented in Fig. 16. ADC interface and PWM block are also assigned to the PL part of the ZYNQ-7000 SoC.

Based on the iteration speed, the digital system for ME can be divided into three subsystems. The first one iterates at 1 $\mathrm{MHz}$, which contains the real-time motor model and resolver emulator. The second one iterates at $400 \mathrm{kHz}$ system, consisting of MPC, ADC, and PWM generation. The third one is the $200 \mathrm{~Hz}$ system, which is for the communication between the SoC and the PC laptop. Those three subsystems are running in parallel with each other. The sequence of operation for the $1 \mathrm{MHz}$ and $400 \mathrm{kHz}$ subsystems is presented in Fig. 17. The motor model update is concurrent with the resolver emulation. The ADC for the resolver emulation has significant latency; therefore, it is done in parallel with the resolver computation and the DAC. This way, the $1 \mathrm{MHz}$ iteration speed is achieved at the price of slightly longer latency. As for the $400 \mathrm{kHz}$ subsystem, at the very start of the $2.5 \mu$ s control period, the duty cycle calculated during the last control period is loaded into the PWM generation block. Also, the ADC for the phase current and DC bus voltage starts at the beginning of the $2.5 \mu$ s control period. When the conversion is finished, then the MPC and integral (I) controller update are initiated. The newly calculated duty cycle is applied at the start of the next control cycle.

For PL computation core design, the workflow in Fig. 1 is followed. One critical decision to make is whether to use floating-point or fixed-point arithmetic. The main advantage of floating-point data type is the ease of implementation, and the PL of ZYNQ-7000 SoC also supports floating-point data types. However, since both the real-time motor model and MPC require lots of computation and low latency, the full floating-point implementation would exceed the limit of on-chip resources and timing requirement. Thus in this application, the
TABLE V

COMPARISON OF ReSOURCE UtiLIZATION AND LATENCY FOR OnLine Motor Model Block

\begin{tabular}{lccc}
\hline \hline & $\begin{array}{l}\text { W/ code style adaption } \\
\text { and pipelining }\end{array}$ & $\begin{array}{l}\text { W/O code style } \\
\text { adaption }\end{array}$ & $\begin{array}{l}\text { W/O } \\
\text { pipelining }\end{array}$ \\
\hline Latency & $29 \mathrm{CLK}$ & $41 \mathrm{CLK}$ & 33 CLK \\
DSP & $38 \%$ & $61 \%$ & $38 \%$ \\
BRAM & $17 \%$ & $17 \%$ & $12 \%$ \\
Flip-flop & $5 \%$ & $8 \%$ & $4 \%$ \\
LUT & $2 \%$ & $4 \%$ & $2 \%$ \\
\hline \hline
\end{tabular}

fixed-point data type is used. Xilinx provides a fixed point data type called ap_fixed in the high-level synthesis (HLS) software [24], which makes it easy to use fixed-point data types in the design. As mentioned above, the coding style adaption and pipelining are also crucial to optimizing latency and resource utilization. In Table $\mathrm{V}$, the comparison of resource utilization and latency of the online motor model block with and without code style adaption and pipelining is shown. It can be seen that the code style adaption significantly reduces the resource and clock cycle required for the computation core, while the pipelining improves the latency at the cost of slightly more resource utilization. Once the design in HLS is finished, it can be verified using the workflow shown in Fig. 1.

For the sequential logic blocks, the PWM generation block consists of several counters and logic gates, designed using library blocks from the System Generator for DSP toolbox in Simulink. However, the ADC interface requires careful design. There are five physical quantities in the ME application that need to be sampled at $400 \mathrm{kHz}$ rate. Three-phase current and two DC bus voltages, one for ME, another one for IUT. To reduce the latency, four external ADC ICs are utilized. Three of them are MAX1304, one for each phase current. The other ADC IC is ADS7865, for two DC bus voltage sensing. Since the timing requirement is much higher than the APF, each $\mathrm{ADC}$ IC can have a dedicated 12-bit parallel data bus and control bus to execute the conversion concurrently. This way, the conversion for all five quantities can be finished within $1.25 \mu \mathrm{s}$.

\section{B. Verification \& Performance Analysis}

The clock speed for PL (200 MHz) and PS (666.7 MHz) is kept the same as in the APF application. The motor model and resolver emulator are iterating at $1 \mathrm{MHz}$. Simultaneously, the current sensing and current controller are running at $400 \mathrm{kHz}$. The latency result shown in Fig. 17 for each task is highly satisfactory, which is achieved since all the intensive computation is executed in PL, and the pipelining technique improves the clock speed. The results also demonstrate the effectiveness of the proposed methodology. If all the computation cores were to be implemented in PS, the additional time it takes to transmit the data between PL and PS could make the whole execution time longer than the control period, let alone the slower computation of PS. As indicated in the figure, the most timeconsuming task is the ADC for current sensing. According to the timing analysis, the system's slowest path still has $0.17 \mathrm{~ns}$ 


\begin{tabular}{|l|r|r|r|}
\hline Resource & Utilization & Available & Utilization \% \\
\hline LUT & 7558 & 46200 & 16.36 \\
\hline LUTRAM & 797 & 14400 & 5.53 \\
\hline FF & 10792 & 92400 & 11.68 \\
\hline BRAM & 62 & 95 & 65.26 \\
\hline DSP & 89 & 160 & 55.62 \\
\hline IO & 77 & 150 & 51.33 \\
\hline BUFG & 2 & 32 & 6.25 \\
\hline
\end{tabular}

Fig. 18. ZYNQ-7000 resource utilization report for ME.

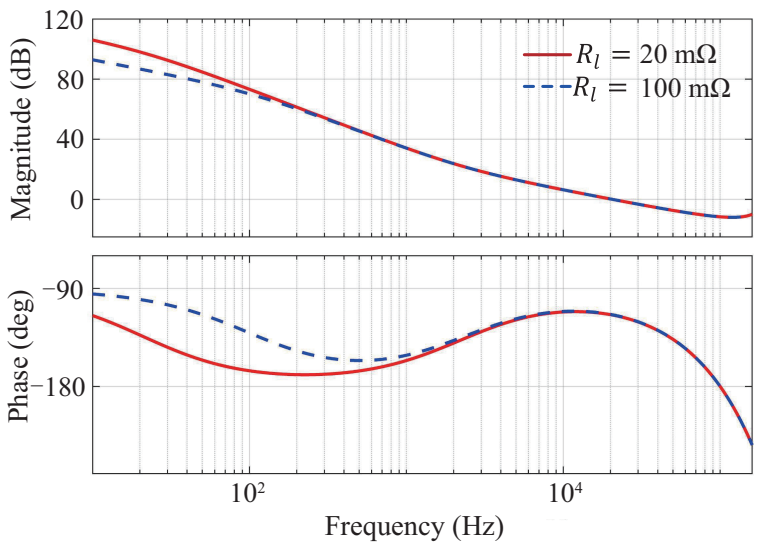

Fig. 19. Compensated open loop response.

slack, and there is no hold violation. If the traditional FPGA design method were to be used, this high timing requirement would be difficult to meet. For resource utilization, the $\mathrm{I} / \mathrm{O}$ pins and DSP core are the most utilized resource at $51.33 \%$ and $55.62 \%$, respectively. This is mainly due to the various peripheral circuits like ADC and DAC needed for sensing and resolver emulation and intense computation of the model predictive control and motor model. It is worth mentioning that the utilization of BRAM is relatively high at $65.26 \%$. However, this is mainly consumed by the Integrated Logic Analyzer (ILA) for debugging purposes. Once development is finished, the BRAM space could be freed up for other use, such as integrating the more accurate and complicated finite element analysis (FEA) based motor model. All other resource utilization rates are relatively low, as shown in Fig. 18.

\section{Controller Analysis \& Experimental Results}

With the controller structure in Fig. 15 and very short sampling time of $2.5 \mu \mathrm{s}, 20 \mathrm{kHz}$ control bandwidth can be achieved, as represented in the compensated open-loop response with different parasitic resistance value in Fig. 19. Furthermore, to improve the disturbance rejection response during the switching transient of IUT, the gate stitching modulation strategy [23] is proposed to achieve accurate IUT terminal voltage feedforward. The proposed modulation scheme takes advantage of the inherent logic gates in FPGA to achieve the function efficiently. Fig. 20 shows the comparison of frequency responses of $Y_{\mathrm{dr}}(\mathrm{s})$ $=i_{\mathrm{x}}(\mathrm{s}) / v_{\mathrm{x}}(\mathrm{s})$ with and without the pulse voltage feedforward. Apparently, without the gate stitching, the disturbance rejection

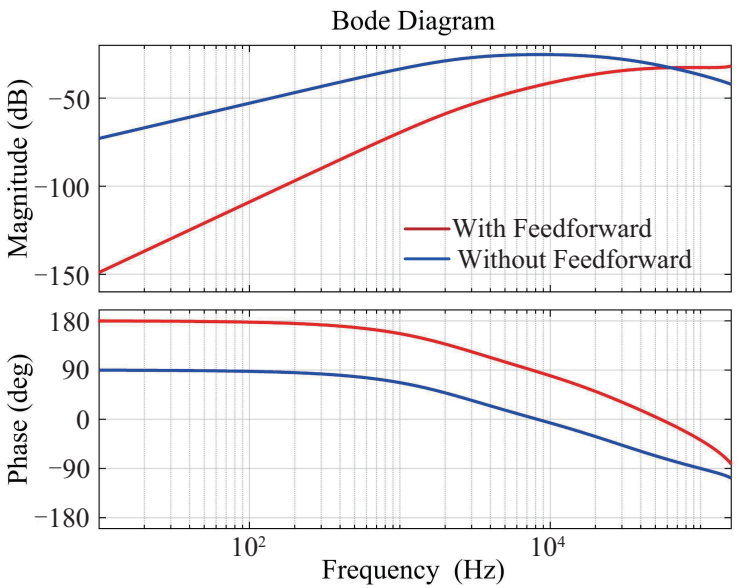

Fig. 20. Disturbance rejection response $Y_{\mathrm{dr}}=i_{\mathrm{x}}(\mathrm{s}) / v_{\mathrm{x}}(\mathrm{s})$ of the MPC with and without the IUT pulse voltage feedforward.

response would deteriorate to the blue trace during the switching transients of IUT. More details of the controller design and analysis can be found in our previous work [23].

Full-scale experiments have been carried out to demonstrate the proposed high bandwidth ME system. The experimental results are given in Fig. 18 where the current reference from the motor model is compared with the actual current in the system. The FFT results are also included to evaluate the current controller tracking performance. The current feedback matches the current reference very well, up to twice the switching frequency of IUT $(20 \mathrm{kHz})$. The matching of the harmonic order is important since this is related to the motor torque ripple order, and the inverter experiences the exact conditions as with a real motor. Moreover, the current tracking error does not vary much with the phase current amplitude, as shown in Fig. 21(a) and Fig. 21(b). Therefore, it is safe to say that even under high phase current operations, the current tracking error would still be maintained at a relatively low level. The high-frequency ripple current caused by the $10 \mathrm{kHz}$ switching of IUT can be accurately emulated in the system. Overall, the proposed ME achieves control bandwidth of $20 \mathrm{kHz}$ [23].

Compared with the existing ME solutions [25], [26], the proposed system provides the high bandwidth current emulation capability up to several times the switching frequency of IUT without the need for complicated power stage topology and inductor design. The FPGA platform and the newly proposed design methodology are the critical enablers for this achievement.

\section{VI.CONCLUSION}

This paper presents a FPGA design methodology for rapid prototyping in power electronics applications. The proposed method utilizes the HLS tool and Simulink code generation blockset to minimize the need for HDL programming during the system design and expedite the verification process while not compromising the FPGA platform's performance. High FPGA clock speed (200 MHz) and low control latency are achieved with reasonable resource consumption. The methodology applies to FPGA-based digital systems available from 

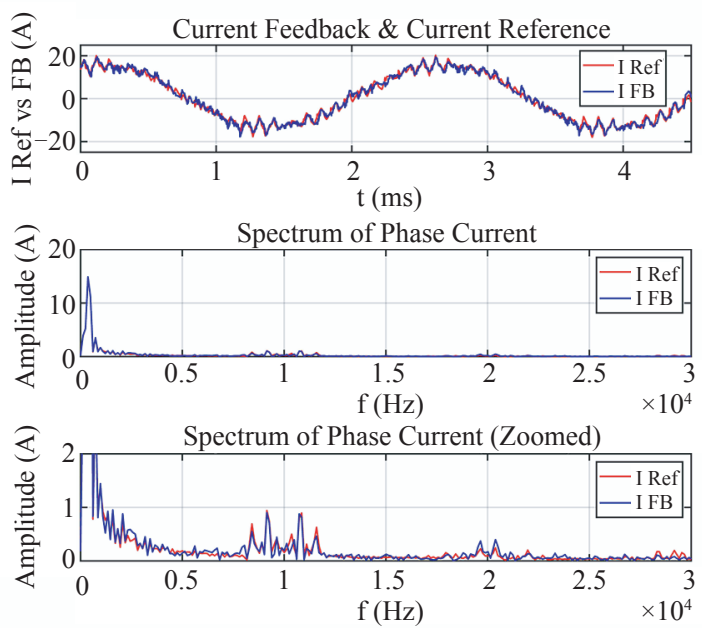

(a) 15 A Operation
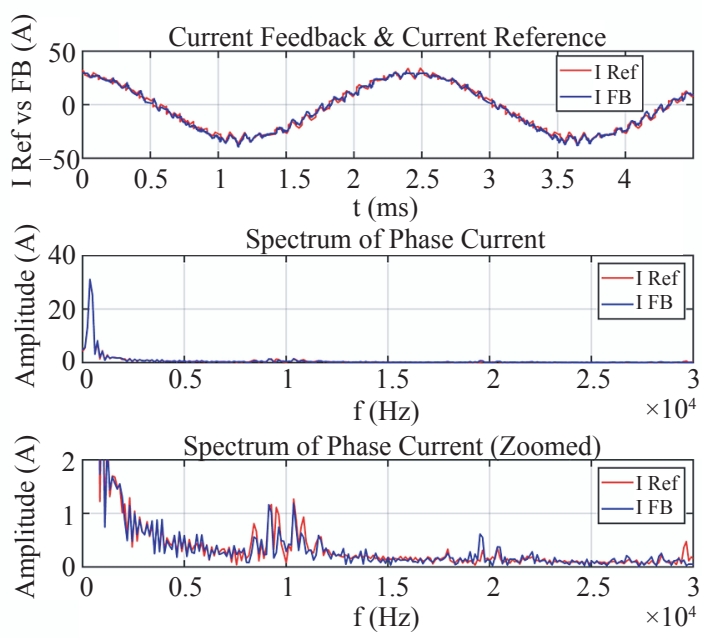

(b) 35 A Operation

Fig. 21. Comparison between current reference and current feedback at $400 \mathrm{~Hz}$ (6000 rpm) with FFT analysis.

different manufacturers for various power electronics applications. Two ultra high bandwidth power electronics implementation examples are discussed in the paper to demonstrate this design method's effectiveness. The first example is an interleaved high bandwidth active power filter (APF) utilizing SiC MOSFET switching at $100 \mathrm{kHz}$. The second one is a high bandwidth motor emulator (ME) based on a SiC power converter with control bandwidth over $20 \mathrm{kHz}$. The two examples have different controller structures and control period requirements, which introduces differences in the implemented digital system structures. Experimental results of two examples demonstrate the effectiveness of the proposed methodology based on the two key performance indicators of clock speed and resource utilization for a FPGA-based embedded system.

\section{ACKNOWLEDGEMENT}

The authors acknowledge Li Yang and Dhrubo Rahman's support for their contribution to the power stage hardware design.

\section{REFERENCES}

[1] T. Kahl and S. Dieckerhoff, "Comparison of FPGA- and microcontrollerbased control of a high-dynamic power electronic converter," in Proceedings of 2017 IEEE 18th Workshop on Control and Modeling for Power Electronics (COMPEL), Stanford, CA, USA, Jul. 2017, pp. 1-6.

[2] Z. Ma, J. Gao, and R. Kennel, "FPGA implementation of a hybrid sensorless control of SMPMSM in the whole speed range," in IEEE Transactions on Industrial Informatics, vol. 9, no. 3, pp. 1253-1261, Aug. 2013.

[3] S. Lucia, D. Navarro, Ó. Lucía, P. Zometa, and R. Findeisen, "Optimized FPGA implementation of model predictive control for embedded systems using high-level synthesis tool," in IEEE Transactions on Industrial Informatics, vol. 14, no. 1, pp. 137-145, Jan. 2018.

[4] W. Tu, G. Luo, Z. Chen, C. Liu, and L. Cui, "FPGA implementation of predictive cascaded speed and current control of PMSM drives with two-time-scale optimization," in IEEE Transactions on Industrial Informatics, vol. 15, no. 9, pp. 5276-5288, Sept. 2019.

[5] S. Wendel, A. Dietz, and R. Kennel, "FPGA based finite-set model predictive current control for small PMSM drives with efficient resource streaming," in Proceedings of 2017 IEEE International Symposium on Predictive Control of Electrical Drives and Power Electronics (PRECEDE), Pilsen, Czech Republic, Sept. 2017, pp. 66-71.

[6] T. J. Vyncke, S. Thielemans, and J. A. Melkebeek, "Finite-set model based predictive control for flying-capacitor converters: cost function design and efficient FPGA implementation," in IEEE Transactions on Industrial Informatics, vol. 9, no. 2, pp. 1113-1121, May 2013.

[7] Z. Zhang, F. Wang, T. Sun, J. Rodríguez, and R. Kennel, "FPGA-based experimental investigation of a quasi-centralized model predictive control for back-to-back converters," in IEEE Transactions on Power Electronics, vol. 31, no. 1, pp. 662-674, Jan. 2016.

[8] E. Lupon, S. Busquets-Monge, and J. Nicolas-Apruzzese, "FPGA implementation of a PWM for a three-phase DC-AC multilevel activeclamped converter," in IEEE Transactions on Industrial Informatics, vol. 10, no. 2, pp. 1296-1306, May 2014.

[9] J. Holtz, M. Höltgen, and J. O. Krah, "A space vector modulator for the high-switching frequency control of three-level SiC inverters," in IEEE Transactions on Power Electronics, vol. 29, no. 5, pp. 2618-2626, May 2014.

[10] S. Ji, M. Laitinen, X. Huang, J. Sun, W. Giewont, F. Wang, and L. M. Tolbert, "Short-circuit characterization and protection of $10 \mathrm{kV} \mathrm{SiC}$ MOSFET," in IEEE Transactions on Power Electronics, vol. 34, no. 2, pp. $1755-1764$, Feb. 2019.

[11] M. Hartmann, S. D. Round, H. Ertl, and J. W. Kolar, "Digital current controller for a $1 \mathrm{MHz}, 10 \mathrm{~kW}$ three-phase VIENNA rectifier," in IEEE Transactions on Power Electronics, vol. 24, no. 11, pp. 2496-2508, Nov. 2009.

[12] G. Martin and G. Smith, "High-level synthesis: Past, present, and future," in IEEE Design \& Test of Computers, vol. 26, no. 4, pp. 18-25, Jul.-Aug. 2009.

[13] E. Guerrero, E. Guzmán, J. Linares, A. Martínez, and G. Guerrero, "FPGA-based active disturbance rejection velocity control for a parallel DC/DC buck converter-DC motor system," in IET Power Electronics, vol. 13, no. 2, pp. 356-367, 2020.

[14] D. Navarro, Ó. Lucía, L. A. Barragán, I. Urriza, and Ó. Jiménez, "High-level synthesis for accelerating the FPGA implementation of computationally demanding control algorithms for power converters," in IEEE Transactions on Industrial Informatics, vol. 9, no. 3, pp. 13711379, Aug. 2013.

[15] O. Gulbudak and E. Santi, "FPGA-based model predictive controller for direct matrix converter," in IEEE Transactions on Industrial Electronics, vol. 63, no. 7, pp. 4560-4570, Jul. 2016.

[16] R. Wiśniewski, G. Bazydło, P. Szcześniak, and M. Wojnakowski, "Petri net-based specification of cyber-physical systems oriented to control direct matrix converters with space vector modulation," in IEEE Access, vol. 7, pp. 23407-23420, Feb. 2019.

[17] M. Hamouda, H. F. Blanchette, K. Al-Haddad, and F. Fnaiech, "An 
efficient DSP-FPGA-based real-time implementation method of SVM algorithms for an indirect matrix converter," in IEEE Transactions on Industrial Electronics, vol. 58, no. 11, pp. 5024-5031, Nov. 2011.

[18] "Vivado Design Suite User Guide: High-Level Synthesis," 2018. [Online]. Available: https://www.xilinx.com/support/documentation/ swmanuals/xilinx2018 3/ug902-vivado-high-level-synthesis.pdf

[19] "Vivado Design Suite Tutorial: Model-Based DSP Design Using System Generator," 2020. [Online]. Available: https://www.xilinx.com/support documentation/sw'manuals/xilinx2020'1/ug948-vivado-sysgen-tutorial. pdf

[20] M. A. Awal, Y. Luo, D. Rahman, L. Yang, W. Yu, and I. Husain, "SiC based interleaved voltage source converter for active power filter applications," in Proceedings of 2020 IEEE Applied Power Electronics Conference and Exposition (APEC), New Orleans, LA, USA, 2020.

[21] R. Peña-Alzola, M. Liserre, F. Blaabjerg, R. Sebastián, J. Dannehl, and F. W. Fuchs, "Analysis of the passive damping losses in LCL-filter-based grid converters," in IEEE Transactions on Power Electronics, vol. 28, no. 6, pp. 2642-2646, Jun. 2013.

[22] J. Dannehl, F. W. Fuchs, S. Hansen, and P. B. Thøgersen, "Investigation of active damping approaches for PI-based current control of gridconnected pulse width modulation converters with LCL filters," in IEEE Transactions on Industry Applications, vol. 46, no. 4, pp. 1509-1517, Jul. 2010.

[23] Y. Luo, M. A. Awal, W. Yu, and I. Husain, "FPGA-based high bandwidth motor emulator for interior permanent magnet machine utilizing $\mathrm{SiC}$ power converter," in IEEE Journal of Emerging and Selected Topics in Power Electronics, vol. 9, no. 4, pp. 4340-4353, Aug. 2021.

[24] A. Finnerty and H. Ratigner, "Reduce Power and Cost by Converting from Floating Point to Fixed Point," 2020. [Online]. Available: https:// www.xilinx.com/support/documentation/white papers/wp491-floatingto-fixed-point.pdf

[25] S. Grubic, B. Amlang, W. Schumacher, and A. Wenzel, "A high performance electronic hardware-in-the-loop drive-load simulation using a linear inverter (LinVerter)," in IEEE Transactions on Industrial Electronics, vol. 57, no. 4, pp. 1208-1216, Apr. 2010.

[26] D. Uebener and J. BÖcke, "Application of an e-machine emulator for power converter tests in the development of electric drives," in Proceedings of EEVC 2012 - European Electric Vehicle Congress, Brussels, Belgium, 2012.

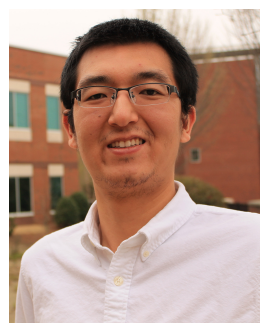

Yukun Luo received the B.S. degree in applied physics from the Huazhong University of Science and Technology, Wuhan, China, in 2015 and the M.S. degree in electrical engineering from the North Carolina State University, Raleigh, NC, USA, in 2017. He is currently working toward the Ph.D. degree with the FREEDM Systems Center, North Carolina State University, Raleigh, NC, USA. His research interests include design of embedded systems on FPGAs for power electronic systems and

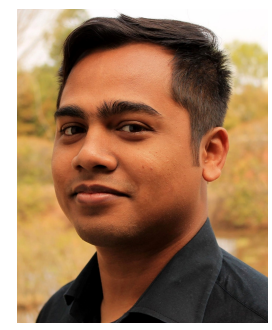

M A Awal received the Ph.D. degree in electrical engineering from North Carolina State University, Raleigh, NC, USA, in 2020. He is currently working as a Controls Engineer in the medium voltage $R \& D$ team at Danfoss Drives. He also serves as a Visiting Scientist at the FREEDM NSF Research Center.

His research interests include design and control of power electronics converters and systems with applications to microgirds, grid interfaces, variable speed drives, and electric automotive systems.

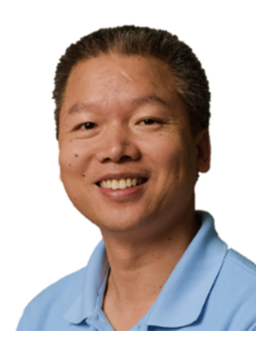

Wensong Yu received the M.S. degree from the Central China University of Science and Technology, and the Ph.D. degree from the South China University of Technology, China, in 1995 and 2000, respectively, both in mechanical and electrical engineering. From 2006 to 2013, he was a Postdoctoral Researcher, Research Scientist, and Research Assistant Professor at the Bradley Department of Electrical and Computer Engineering at Virginia Polytechnic Institute and State University, Blacksburg, VA, USA. Since 2013, he has been with the Department of Electrical and Computer Engineering at North Carolina State University, Raleigh, NC, USA, as a Research Associate Professor. His current research interests are high-frequency solid-state transformer, advanced soft-switching technique, wide bandgap device applications, ultra-high efficiency inverter, high-voltage power conversion and protection, $\mathrm{SiC}$ electric vehicle traction drive, distributed energy storage devices, and green energy grid infrastructure.

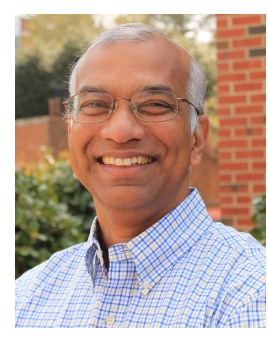

Iqbal Husain is the Director of the FREEDM NSF Engineering Center and the ABB Distinguished Professor in the Department of Electrical \& Computer Engineering at North Carolina State University, Raleigh, NC. Prior to joining NC State, he was at the University of Akron where he built a successful power electronics and motor drives program. He was a Visiting Professor at Oregon State University, Corvallis, OR in 2001. Dr. Husain's expertise is in the areas of power electronics, electric machines, motor drives, and system controls. His research is also focused on power electronics and drives integration into power and transportation systems. The primary applications of his work are in the transportation, automotive, aerospace and power industries. Dr. Husain has also developed and taught many graduate and undergraduate courses in the power and energy area and published the textbook Electric and Hybrid Vehicles: Design Fundamentals.

Dr. Husain received his Ph.D. degree in electrical engineering from Texas A\&M University in 1993. He received the 2006 SAE Vincent Bendix Automotive Electronics Engineering Award, the 2004 College of Engineering Outstanding Researcher Award, the 2000 IEEE Third Millennium Medal and the 1998 IEEE-IAS Outstanding Young Member award. He became an IEEE Fellow in 2009. He is the past Editor-in-Chief of the IEEE Electrification Magazine. 